\title{
The development of a provincial
}

\section{multidisciplinary framework of consensus-based standards for Point of Care Ultrasound at the University of Saskatchewan}

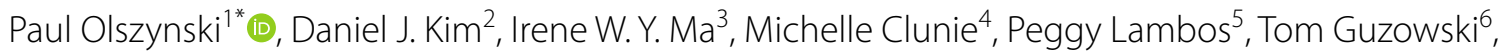
Matthew Butz ${ }^{7}$ and Brent Thoma'

\begin{abstract}
Objectives: The development and adoption of Point-of-Care Ultrasound (POCUS) across disciplines have created challenges and opportunities in implementing training and utilization standards. Within the context of a large, geographically disparate province, we sought to develop a multidisciplinary POCUS framework outlining consensusbased standards.

Methods: A core working group of local POCUS leaders from Anesthesia, Emergency Medicine, Family Medicine, Intensive Care, Internal Medicine, Pediatrics, and Trauma, in collaboration with western Canadian colleagues, developed a list of key domains for the framework along with a range of potential standards for each area. The members of the working group and the registrants for a multidisciplinary Roundtable discussion at the University of Saskatchewan's annual POCUS conference (SASKSONO19, Saskatoon, Saskatchewan, March 2nd, 2019) were invited to complete a survey on POCUS standards for each domain. The survey results were presented to and discussed by participants at the Roundtable discussion at SASKSONO19 who reached consensus on modified standards for each domain. The modified standards were considered for endorsement by all conference attendees using an audience-response system.

Results: The working group proposed standards in eight domains: scope of use, credentialing and privileges, documentation, quality assurance, leadership and governance, teaching, research, and equipment maintenance. Consensus on modified standards was achieved in the 18 participant Roundtable. Each standard was then endorsed by $>90 \%$ of conference respondents.

Conclusion: The resulting framework will inform the utilization of POCUS within Saskatchewan. Both this process and its outcomes could inform the development of multidisciplinary POCUS standards within other jurisdictions.
\end{abstract}

Keywords: Point of Care Ultrasound, Framework, Standards, Multidisciplinary, Consensus, Quality assurance

\footnotetext{
*Correspondence: p.olszynski@usask.ca

1 Department of Emergency Medicine, University of Saskatchewan,

Saskatoon, Canada

Full list of author information is available at the end of the article
} 


\section{Introduction}

Point of Care Ultrasound (POCUS) is defined as "diagnostic or procedural guidance ultrasound that is performed by a clinician during a patient encounter to help guide the evaluation and management of that patient." [1] It can impact a range of patient-related outcomes including diagnostic accuracy [2-6], time to diagnosis $[7,8]$, time to definitive management [8-10], procedural safety [11-16], decreased complications [15, 16], morbidity [17, 18], and mortality [19]. Several disciplines have developed guidelines and standards for the use of POCUS in clinical practice [12, 13, 20-25]. These POCUS standards offer guidance for evidence-based application, general training requirements, documentation standards, quality assurance, and equipment maintenance. As more healthcare disciplines adopt POCUS, it will be increasingly common for healthcare providers to exchange POCUS findings during transitions in care.

POCUS is used by a wide range of disciplines. While individual applications and specific uses of POCUS vary between disciplines, domains such as scope of use, training, and governance are similar and amenable to forming a common standard [26]. Multidisciplinary POCUS collaboration and consensus on standards may help ensure consistent patient care and support optimal POCUS training within our clinical context. The establishment of a common framework of standards for POCUS users may also increase the quality of POCUS scans and improve communication regarding patient findings. Given that some disciplines have more extensive experience in the integration of POCUS into clinical practice, we anticipate that the creation of a multidisciplinary, mutually agreed upon POCUS framework will facilitate the spread of best practices between disciplines.

We sought to develop and build consensus around a local multidisciplinary framework of consensus-based POCUS standards. We believe that this process could be utilized by other institutions and/or health associations to develop their own set of multidisciplinary POCUS standards.

\section{Methods}

Collectively, we developed an iterative, four-part process to draft and build consensus around a multidisciplinary framework of POCUS standards (Fig. 1). Our process was informed in part by similar consensus-based processes carried out by colleagues in other fields [27, 28]. A Research Ethics Board exemption was sought and obtained from the University of Saskatchewan's Research Ethics Board (BEH 957). Participation in any of the aspects of the framework process was voluntary and consent was implied through participation.

First, a working group of local POCUS leaders from Anesthesia, Critical Care, Emergency Medicine, Family

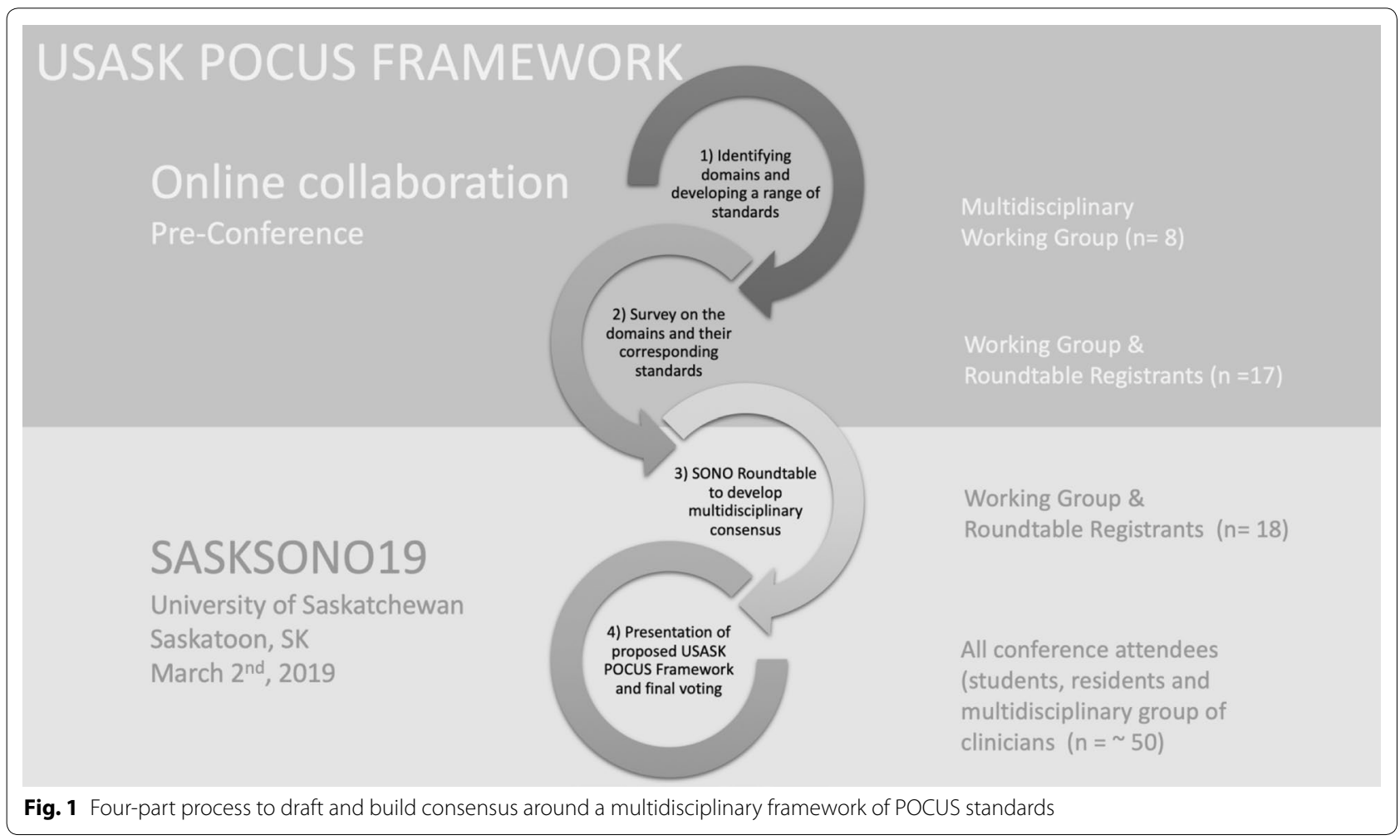


Medicine, Internal Medicine, Pediatrics, and Trauma was identified based upon their leadership roles in the adoption of POCUS within their discipline in Saskatchewan and subsequently invited to participate via email invitations. The expertise within this group was buttressed through collaboration with western Canadian POCUS leaders that had been invited to present at the University of Saskatchewan's annual POCUS Conference (SASKSONO19, Saskatoon, Saskatchewan, March 2nd, 2019). We reviewed other previously published domain-specific POCUS guidelines to develop a list of key domains for a multidisciplinary framework [12, 13, 20-25]. Once the domains had been determined, a range of potential standards for each domain was proposed by one working group member (PO). These potential standards were disseminated to the working group who reviewed and edited them until it was felt that they represented a reasonable possible standard that could be considered (Additional file 1: Appendix S1).

Second, members of the working group along with the registrants for a Roundtable discussion at SASKSONO19 were invited to complete a survey of the aforementioned proposed standards. Roundtable participants were required to be clinicians who use POCUS regularly in their practice. The survey was hosted on Google Forms (Google, Mountain View, CA) and asked each participant to indicate their preferred standard from the range of potential standards developed for each domain. 'Other' was also an option within each domain and, when selected, participants were invited to input alternative standards in free text.

Third, the results of this survey were presented to the participants who attended the Roundtable discussion at SASKSONO19. The results for each domain, including both the vote totals for the range of standards and the standards that were proposed within the survey were reviewed. Where there was a lack of clear consensus on one of the proposed standards, a detailed discussion occurred with the goal of drafting a standard for each domain that all the participants could support. Ultimately, consensus was achieved within the working group on a single standard for each domain.

Finally, the single modified standard supported by the members of the Roundtable discussion for each of the domains was reviewed by the conference attendees at the final plenary session of the conference. The rationale for each standard was explained, and conference attendees (including students, residents, and clinicians from multiple disciplines) indicated whether or not they supported each proposed standard using an audience-response system (Mentimeter, Stockholm, Sweden). We defined consensus a priori as an endorsement by $>80 \%$ of the respondents in each of our iterative consensus process (working group members, roundtable participants and conference attendees).

\section{Results}

The eight working group participants developed a list of nine domains to be addressed in the framework (scope of use, credentialing and privileges, documentation, quality assurance, leadership and governance, teaching, research, and equipment maintenance) and proposed three potential standards for each (Additional file 1: Appendix S1).

The survey of the potential standards sent to the working group members and Roundtable registrants was completed by 17 clinicians (Table 1). Survey results (Table 2) revealed unanimous agreement in some domains (e.g., Scope of Use: The appropriate application of POCUS should be defined by individual disciplines and be used whenever supported by reasonable evidence) whereas there were a range of opinions and suggestions on other domains (e.g., Documentation standards). The standards that were suggested for each domain are outlined in Additional file 2: Appendix S2.

The survey results (Table 2) were presented to the 18 attendees at the Roundtable discussion (Table 1). For each domain, informal consensus was sought and obtained on one of the initial proposed standards or a new standard drafted within the session. Several items within the framework generated significant discussion at the Roundtable, likely stemming from the variable stages of development and utilization of POCUS by the multidisciplinary group of stakeholders.

Image capture proved particularly controversial as it related to the domains of documentation in the medical record and quality assurance while the domain of credentials and privileges also required significant discussion to reach consensus. While a standard requiring documentation of POCUS findings in the form of a structured clinical note was widely accepted, there was extensive

\begin{tabular}{lllll}
$\begin{array}{l}\text { Table } 1 \text { Discipline } \\
\text { participants }\end{array}$ & working group & and roundtable \\
\hline Participants & $\begin{array}{l}\text { Working group } \\
(\boldsymbol{n}=\mathbf{8})\end{array}$ & $\begin{array}{l}\text { Roundtable } \\
(\boldsymbol{n}=\mathbf{1 8})\end{array}$ \\
\hline Saskatchewan-based & EM & 3 & EM & 7 \\
& IM & 1 & IM & 1 \\
& Anesthesia & 1 & Anesthesia & 3 \\
& Pediatrics & 1 & Critical care & 1 \\
& & & Pediatrics & 1 \\
& & & FM & 3 \\
Invited from out-of-province & EM & 1 & EM acute care & 1 \\
& IM & 1 & & 1 \\
\hline
\end{tabular}

$E M$ emergency medicine, $I M$ internal medicine, $F M$ family medicine, $N P$ nurse practitioner 
Table 2 Results of the pre-conference survey of the potential standards ${ }^{a}$ for each domain in order of preference

\begin{tabular}{|c|c|c|}
\hline Domain & Preferred option ${ }^{a}$ & $\begin{array}{l}\text { Support } \\
(n=17) \\
(\%)\end{array}$ \\
\hline Scope of use & $\begin{array}{l}\text { 1. The appropriate application of POCUS should be defined by individual specialties/disciplines } \\
\text { and be used whenever supported by reasonable evidence }\end{array}$ & 100 \\
\hline \multirow[t]{3}{*}{ Credentials and privileges } & $\begin{array}{l}\text { 1. Departments should define specific credentials that are required to receive privileges to use } \\
\text { POCUS }\end{array}$ & 35 \\
\hline & $\begin{array}{l}\text { 2. No credentials or additional privileges should be required for the use of POCUS; its use should } \\
\text { be up to the clinician similar to any other aspect of the clinical assessment }\end{array}$ & 25 \\
\hline & $\begin{array}{l}\text { 3. Any additional training required (and associated privileges) to use POCUS should be determined } \\
\text { on a case-by-case basis by each department }\end{array}$ & 18 \\
\hline \multirow[t]{3}{*}{ Documentation } & $\begin{array}{l}\text { 1. POCUS findings should be documented, and images captured when they play a significant role } \\
\text { in patient care decisions }\end{array}$ & 59 \\
\hline & $\begin{array}{l}\text { 2. POCUS findings should be documented, and all images should be captured for inclusion in the } \\
\text { patient's medical record }\end{array}$ & 25 \\
\hline & $\begin{array}{l}\text { 3. POCUS should be documented in the same way as physical exam findings as part of the overall } \\
\text { clinical assessment }\end{array}$ & 18 \\
\hline \multirow[t]{2}{*}{ Quality assurance } & $\begin{array}{l}\text { 1. An audit of POCUS should be coordinated by any groups utilizing POCUS. Review of images, } \\
\text { when available, is strongly encouraged }\end{array}$ & 88 \\
\hline & $\begin{array}{l}\text { 2. POCUS use should include image capture and all images must be reviewed for quality assurance } \\
\text { purposes. }(12 \%)\end{array}$ & 12 \\
\hline \multirow[t]{2}{*}{ Leadership and governance } & 1. Multidisciplinary committee with representatives from each specialty/discipline using POCUS & 59 \\
\hline & 2. Each specialty/discipline oversees its own use (41\%) & 41 \\
\hline \multirow[t]{3}{*}{ Teaching } & 1. POCUS education can be provided by those with privileges recognized by their department & 41 \\
\hline & $\begin{array}{l}\text { 2. POCUS education can be provided only by those with specific credentials as determined by a } \\
\text { multidisciplinary POCUS committee }\end{array}$ & 35 \\
\hline & 3. POCUS education can be provided by clinicians without specific credentials & 6 \\
\hline \multirow[t]{2}{*}{ Research } & $\begin{array}{l}\text { 1. Concerted and coordinated efforts to maximize research productivity to help propel USASK as a } \\
\text { leader in POCUS research }\end{array}$ & 59 \\
\hline & 2. Clinical and educational/training research should be encouraged within each department & 41 \\
\hline \multirow[t]{2}{*}{ Equipment support and maintenance } & $\begin{array}{l}\text { 1. Universal standards for POCUS equipment support and maintenance should be coordinated } \\
\text { centrally within SHA }\end{array}$ & 59 \\
\hline & $\begin{array}{l}\text { 2. Standards for POCUS equipment support and maintenance standards should be coordinated by } \\
\text { each institution within SHA }\end{array}$ & 41 \\
\hline
\end{tabular}

${ }^{a}$ Only potential standards which received at least one vote in support are included. The full set of potential standards is included in the survey in Additional file 1: Appendix S1

Roundtable discussion on the capture, storage, and accessibility of POCUS images as they relate to the domains of documentation and quality assurance. Those in favor of image capture saw it as a best practice that should be regularly used at major teaching/training institutions to facilitate indirect supervision of trainee scans as well as quality assurance [29]. Further, it was felt that the ability to share images and/or videos in real time would add value to patient care in cases where a consultant could review pertinent POCUS findings in real time. It was highlighted that in some cases, this may make the difference between a patient staying at a regional site or being transferred to a referral centre. On the other hand, some participants countered that in certain instances image capture seemed an unreasonable requirement.

Rural and regional stakeholders noted that it was not feasible nor cost-effective to implement image capture middleware in every rural centre using POCUS at this time.

It was highlighted that practice audits can be performed by comparing the clinical notes of the POCUS findings with consultative images when these are available. Ultimately, consensus on documentation was reached along with the understanding that the advancement of POCUS technology, and the evolution of POCUS as a distinct imaging modality [21] with its own criteria for image capture, would guide further iterations of the framework.

The consensus built among this group paved the way for broad agreement among the conference attendees. Table 3 outlines the standards endorsed in the plenary session along with the results of the vote totals. Consensus (defined a priori as $>80 \%$ agreement) was reached for the standards under each of the domains. 
Table 3 Consensus domains and plenary session support for each of the standards within the multidisciplinary POCUS framework

\begin{tabular}{|c|c|c|}
\hline Domain & Standard & Support $^{\mathrm{a}}$ \\
\hline 1. Scope of use & $\begin{array}{l}\text { The appropriate application of POCUS should be defined by individual disciplines and be used } \\
\text { whenever supported by reasonable evidence }\end{array}$ & $\begin{array}{l}46 / 48 \\
96 \%\end{array}$ \\
\hline 2. Credentials and privileges & $\begin{array}{l}\text { Disciplines should define specific and evolving required credentials that must be met for their } \\
\text { providers to receive and maintain privileges to use POCUS. These credentials should be consist- } \\
\text { ent with national standards }\end{array}$ & $\begin{array}{l}49 / 50 \\
98 \%\end{array}$ \\
\hline 3. Documentation in the medical record & $\begin{array}{l}\text { POCUS findings should be documented in the patient chart, much like the physical exam find- } \\
\text { ings as part of the overall clinical assessment. When image capture is available, select POCUS } \\
\text { images should be archived and available to support the patient's ongoing care. When image } \\
\text { capture is not available, departments should develop a system to track POCUS to support qual- } \\
\text { ity assurance and the patient's ongoing care }\end{array}$ & $\begin{array}{l}47 / 49 \\
96 \%\end{array}$ \\
\hline 4. Quality assurance & $\begin{array}{l}\text { An audit of POCUS should be coordinated by any groups utilizing POCUS. Review of images, when } \\
\text { available, is strongly encouraged. The details of this process should be determined by each discipline }\end{array}$ & $\begin{array}{l}45 / 49 \\
92 \%\end{array}$ \\
\hline 5. Leadership and governance & $\begin{array}{l}\text { Each discipline should oversee its own use of POCUS. A multidisciplinary committee with repre- } \\
\text { sentatives from each discipline should be formed to collaborate and promote best practices }\end{array}$ & $\begin{array}{l}47 / 50 \\
94 \%\end{array}$ \\
\hline 6. Teaching & $\begin{array}{l}\text { Formal POCUS education and assessment can be provided by those with credentials recognized } \\
\text { by their discipline }\end{array}$ & $\begin{array}{l}50 / 52 \\
96 \%\end{array}$ \\
\hline 7. Research & $\begin{array}{l}\text { Clinical and educational research should be encouraged within each department. There should } \\
\text { be a concerted effort to coordinate research between disciplines to maximize research produc- } \\
\text { tivity to help propel the University of Saskatchewan as a leader in POCUS research }\end{array}$ & $\begin{array}{l}50 / 53 \\
94 \%\end{array}$ \\
\hline 8. Equipment support and maintenance & $\begin{array}{l}\text { Standards for POCUS equipment support and maintenance standards should be coordinated by } \\
\text { each discipline as per current best practices and safety guidelines }\end{array}$ & $\begin{array}{l}47 / 52 \\
90 \%\end{array}$ \\
\hline
\end{tabular}

a Not all conference attendees responded to each of the polls

\section{Discussion}

We have developed a consensus-based multidisciplinary POCUS framework outlining standards for eight domains: scope of use, credentialing and privileges, documentation, quality assurance, leadership and governance, teaching, research, and equipment maintenance. Consensus on modified standards was achieved in the 18 participant Roundtable and endorsed by $>90 \%$ of respondents at the conference.

We anticipate that this framework will be used in two ways. Locally, our working group will present our findings to our Health Association's Provincial Practitioner Advisory Committee as a next step in establishing minimum standards. We expect that these standards will inform the local adoption of discipline-specific guidelines that meet or exceed the requirements we have outlined (our Department of Emergency Medicine has already begun this process). More broadly, we anticipate that our process could be used by health professionals in other jurisdictions to develop their own multidisciplinary framework in a similar consensus-building manner.

Our working group sought to develop a multidisciplinary framework that was both provincial and institutional in application. Foundational to our approach was an understanding that, as per the American Medical Association resolution 802, training and education standards for the use of ultrasound imaging be developed by each physician's respective specialty [30]. Building from this, our goal was to develop a common framework upon which POCUS will continue to thrive within our institution. Other consensus processes described in the literature have been either specialty specific, hospital-based [26], or part of a broader clinical or billing protocol issued by a provincial College [31]. Notably, the Canadian Association of Radiologists recently published a position statement written exclusively by radiologists with no evidence of input from the POCUS community or any other discipline [32]. We believe our process is more pragmatic and has greater legitimacy in that we utilized a collaborative and consensus-based approach that incorporated the perspectives of multiple disciplines and clinical environments.

One area of discussion which was not anticipated within the pre-developed domains was the question of which instances and images (when captured on a middleware platform) should then be exported to the patient's permanent medical record. While all agreed that, when possible, scans that significantly impacted medical-decision making should be documented with images and/or video, questions remain regarding the necessity of recording procedural scans (e.g., central venous catheter placement). In addition, it would seem appropriate to consult patients about their preference as well. A subsequent review of the literature on this topic provided limited guidance. Local audits within the department of Emergency Medicine, comparing the written findings with consultative imaging, have revealed a concordance rate in excess of $90 \%$ and demonstrated appropriate application and integration of POCUS [33]. As such, there were concerns that the adoption of an image capture requirement 
would potentially and unjustifiably delay the uptake of POCUS by providers and for patients with the least access to advanced imaging. Further, given the vast range of POCUS applications, several participants suggested that a "one size fits all" approach is likely not appropriate. It was noted that POCUS findings land along a spectrum of clinical meaning and utility, at one end serving as an extension of the physical exam (consider POCUS for jugular venous pressure or interstitial lung syndrome) and the other performing as diagnostic imaging (consider POCUS for intrauterine pregnancy or lower extremity deep venous thrombosis). This spectrum of utility and impact will need to be kept under consideration as image capture standards evolve. As this was not addressed in our current framework, image capture for the patients' permanent record will need to be determined by both discipline-specific experts as well as our colleagues on the receiving end of transitions in care.

\section{Limitations}

The process of developing the multidisciplinary framework had several limitations. Despite efforts to recruit representatives from all disciplines using POCUS in our province, specialties including Neonatal Critical Care and Physiatry were not involved. Further, although we did have working group representatives from three western Canadian provinces, the majority of our experts were based in one province. Therefore, applicability of our POCUS standards to other jurisdictions will need to be decided on a case-by-case basis. Additionally, we did not grade the strength of each recommendation, as this was not part of our protocol. A significant outstanding question regarding when image capture needs to be used was raised during the Roundtable discussion, but was not addressed through this process. Finally, we believe our multidisciplinary approach would be enriched by having input from colleagues who have traditionally interpreted diagnostic ultrasonography in the fields of cardiology, obstetrics/gynecology, and medical imaging. These specialties were invited to participate in our working group, but unfortunately, there was no response to the invitation.

The adoption of provincial standards will be an iterative process, with members of our working group dedicated to ongoing discussions with our health authority as well as with other colleagues. We plan to reconvene the Roundtable annually at each SASKSONO event to address current standards and evolving issues.

\section{Conclusion}

In conclusion, our multidisciplinary POCUS framework provides a provincial standard upon which each discipline utilizing POCUS can build. It represents one of many initiatives to ensure high-quality use of POCUS that accounts for its use across many different clinical settings (including pre-hospital, the emergency department, the operating room, critical care unit, surgical and medical wards, and outpatient clinics). Locally, it will inform the implementation and utilization of POCUS within the University of Saskatchewan and the Saskatchewan Health Authority. More broadly, this process and its outcomes could be used as a template for the development of multidisciplinary POCUS standards within other jurisdictions.

\section{Supplementary information}

Supplementary information accompanies this paper at https://doi. org/10.1186/s13089-019-0142-7.

Additional file 1. USASK POCUS Framework survey.

Additional file 2. Survey results (comprehensive).

\section{Acknowledgements}

In collaboration with the SASKSONO19 Sono-Roundtable attendees/participants: Faisal Al-Sani, Robert Appleton, Rachit Batta, Kex Cau, Joann Kawchuk, Malcolm Lucy, Dalmar Lynds, Brady Murphy, Quinten Paterson, Rob Peverseff, James Prunell, James Stempien, Krista Wempe, and Rob Woods.

We also thank the attendees of SASKSONO19 for their participation in the audience poll-response system that provided feedback on the proposed standards.

\section{Authors' contributions}

PO and BT contributed to the conception and design of the study, the acquisition of data, the analysis and/or interpretation of data, the drafting of the manuscript, revising the manuscript critically for important intellectual content, and approval of the version of the manuscript to be published. DK contributed to the design of the study, the acquisition of data, the analysis and/or interpretation of data, the drafting of the manuscript, revising the manuscript critically for important intellectual content, and approval of the version of the manuscript to be published. IM contributed to the design of the study, data acquisition and interpretation, the analysis and/or interpretation of data, revised manuscript critically for intellectual content, and approved final manuscript. MC contributed to the design of the study, the acquisition of data, the analysis and/or interpretation of data, revising the manuscript critically for important intellectual content, and approval of the version of the manuscript to be published. PL, TG and MB contributed to the acquisition of data, revising the manuscript critically for important intellectual content, and approval of the version of the manuscript to be published. All authors read and approved the final manuscript.

Funding

Not applicable.

\section{Availability of data and materials}

All data generated or analysed during this study are included in this published article and its Additional files.

\section{Ethics approval and consent to participate}

A Research Ethics Board exemption was sought and obtained from the University of Saskatchewan's Research Ethics Board (BEH 957). Participation in any of the aspects of the framework process was voluntary and consent was implied through participation.

\section{Consent for publication}

Not applicable.

\section{Competing interests}

PO declares that he is a local director and instructor with several POCUS training courses throughout Canada. No recommendations for any specific courses or products are made within this document. DK declares that he is on the 
medical advisory board of Clarius Mobile Health. IM declares no competing interests. All other authors' declare that they have no competing interests.

\section{Author details}

${ }^{1}$ Department of Emergency Medicine, University of Saskatchewan, Saskatoon, Canada. ${ }^{2}$ Department of Emergency Medicine, University of British Columbia, Vancouver, Canada. ${ }^{3}$ Department of Medicine, University of Calgary, Calgary, Canada. ${ }^{4}$ Department of Anesthesia and Perioperative Medicine, University of Saskatchewan, Saskatoon, Canada. ${ }^{5}$ Department of Pediatrics, University of Saskatchewan, Prince Albert, Canada. ${ }^{6}$ Department of Internal Medicine, University of Saskatchewan, Saskatoon, Canada. ${ }^{7}$ Department of Family Medicine, University of Saskatchewan, Regina, Canada.

Received: 3 May 2019 Accepted: 3 October 2019

Published online: 17 October 2019

\section{References}

1. Moore CL, Copel JA (2011) Point-of-Care Ultrasonography. N Engl J Med 364(8):749-757. https://doi.org/10.1056/NEJMra0909487

2. Rubano E, Mehta N, Caputo W, Paladino L, Sinert R (2013) Systematic review: emergency department bedside ultrasonography for diagnosing suspected abdominal aortic aneurysm. Acad Emerg Med 20(2):128-138. https://doi.org/10.1111/acem.12080

3. Burnside PR, Brown MD, Kline JA (2008) Systematic review of emergency physician-performed ultrasonography for lower-extremity deep vein thrombosis. Acad Emerg Med 15(6):493-498. https://doi.org/10.11 11/j.1553-2712.2008.00101.x

4. Long L, Zhao H-T, Zhang Z-Y, Wang G-Y, Zhao H-L (2017) Lung ultrasound for the diagnosis of pneumonia in adults. Medicine (Baltimore) 96(3):e5713. https://doi.org/10.1097/MD.0000000000005713

5. Subramaniam S, Bober J, Chao J, Zehtabchi S (2016) Point-of-care Ultrasound for diagnosis of abscess in skin and soft tissue infections. Acad Emerg Med 23(11):1298-1306. https://doi.org/10.1111/acem.13049

6. Nishijima DK, Simel DL, Wisner DH, Holmes JF (2012) Does this adult patient have a blunt intra-abdominal injury? JAMA 307(14):1517. https ://doi.org/10.1001/jama.2012.422

7. Plummer D, Clinton J, Matthew B (1998) Emergency department ultrasound improves time to diagnosis and survival of ruptured abdominal aortic aneurysm. Acad Emerg Med 5:417

8. Rodgerson JD, Heegaard WG, Plummer D, Hicks J, Clinton J, Sterner $S$ (2001) Emergency department right upper quadrant ultrasound is associated with a reduced time to diagnosis and treatment of ruptured ectopic pregnancies. Acad Emerg Med 8(4):331-336

9. Plummer D, Brunette D, Asinger R, Ruiz E (1992) Emergency department echocardiography improves outcome in penetrating cardiac injury. Ann Emerg Med 21(6):709-712

10. Melniker LA, Leibner E, McKenney MG, Lopez P, Briggs WM, Mancuso CA (2006) Randomized controlled clinical trial of point-of-care, limited ultrasonography for trauma in the emergency department: the first sonography outcomes assessment program trial. Ann Emerg Med 48(3):227-235. https://doi.org/10.1016/j.annemergmed.2006.01.008

11. Practice Guidelines for Central Venous Access (2012) A report by the american society of anesthesiologists task force on central venous access. Anesthesiology 116(3):539-573. https://doi.org/10.1097/ ALN.0b013e31823c9569

12. Soni NJ, Schnobrich D, Mathews BK et al (2019) Point-of-care ultrasound for hospitalists: a position statement of the society of hospital medicine. J Hosp Med. https://doi.org/10.12788/jhm.3079

13. Ma IWY, Arishenkoff S, Wiseman J et al (2017) Internal medicine pointof-care ultrasound curriculum: consensus recommendations from the canadian internal medicine ultrasound (CIMUS) group. J Gen Intern Med 32(9):1052-1057. https://doi.org/10.1007/s11606-017-4071-5

14. Cho J, Jensen TP, Reierson K et al (2019) Recommendations on the use of ultrasound guidance for adult abdominal paracentesis: a position statement of the society of hospital medicine. J Hosp Med 14:E7-E15. https://doi.org/10.12788/jhm.3095

15. Latteri S, Malaguarnera G, Mannino M et al (2017) Ultrasound as point of care in management of polytrauma and its complication. J Ultrasound 20(2):171-177. https://doi.org/10.1007/s40477-017-0252-7
16. Peabody CR, Mandavia D (2017) Deep needle procedures: improving safety with ultrasound visualization. J Patient Saf 13(2):103-108. https //doi.org/10.1097/PTS.0000000000000110

17. Mateer JR, Valley VT, Aiman EJ, Phelan MB, Thoma ME, Kefer MP (1996) Outcome analysis of a protocol including bedside endovaginal sonography in patients at risk for ectopic pregnancy. Ann Emerg Med 27(3):283-289

18. Mitchell D, Leng A, Gardiner S et al (2016) Using point-of-care bedside ultrasound for volume assessment in early shock: an outcome study. Chest 149(4):A148. https://doi.org/10.1016/j.chest.2016.02.154

19. Hans SS, Huang RR (2003) Results of 101 ruptured abdominal aortic aneurysm repairs from a single surgical practice. Arch Surg 138(8):898. https://doi.org/10.1001/archsurg.138.8.898

20. McCormick TJ, Miller EC, Chen R, Naik VN (2018) Acquiring and maintaining point-of-care ultrasound (POCUS) competence for anesthesiologists. Can J Anesth Can d'anesthésie 65(4):427-436. https://doi. org/10.1007/s12630-018-1049-7

21. Guidelines Ultrasound (2017) Emergency, point-of-care and clinical ultrasound guidelines in medicine. Ann Emerg Med 69(5):e27-e54. https://doi.org/10.1016/j.annemergmed.2016.08.457

22. Contra Costa Family Medicine Residency Program. Recommended Curriculum Guidelines for Family Medicine Residents Point of Care Ultrasound. Martinez, CA; 2016. https://www.aafp.org/dam/AAFP/ documents/medical_education_residency/program_directors/Repri nt290D_POCUS.pdf. Accessed 2 Apr 2019

23. Arntfield R, Millington S, Ainsworth $C$ et al (2014) Canadian recommendations for critical care ultrasound training and competency. Can Respir J 21(6):341-345. https://doi.org/10.1155/2014/216591

24. Shefrin AE, Warkentine F, Constantine E et al (2019) Consensus core point-of-care ultrasound applications for pediatric emergency medicine training. AEM Educ Train. https://doi.org/10.1002/aet2.10332

25. Henneberry RJ, Hanson A, Healey A et al (2012) Use of point of care sonography by emergency physicians. CJEM 14(02):106-112. https:// doi.org/10.2310/8000.CAEPPS

26. Mathews B, Zwank M (2017) Hospital medicine point of care ultrasound credentialing: an example protocol. J Hosp Med 12(9):767-772. https://doi.org/10.12788/jhm.2809

27. Woods RA, Artz JD, Carrière B et al (2017) CAEP 2016 academic symposium on education scholarship: training our future clinician educators in emergency medicine. Can J Emerg Med 19(S1):S1-S8. https://doi. org/10.1017/CEM.2017.41

28. Humphrey-Murto S, Varpio L, Gonsalves C, Wood TJ (2017) Using consensus group methods such as Delphi and Nominal Group in medical education research. Med Teach 39(1):14-19. https://doi. org/10.1080/0142159X.2017.1245856

29. Lewis D, Rang L, Kim D et al (2019) Recommendations for the use of point-of-care ultrasound (POCUS) by emergency physicians in Canada. CJEM. https://doi.org/10.1017/cem.2019.392

30. American Medical Association. Privileging for Ultrasound Imaging H-230.960. https://policysearch.ama-assn.org/policyfinder/detail/Ultra soundimaging? uri=\%2FAMADoc\%2FHOD.xml-0-1591.xml. Published 2010. Accessed 22 Aug 2019

31. College of Physicians and Surgeons of Alberta. Diagnostic Imaging Modality Application for Physician Approval Ultrasound Restricted Approval; 2019. http://www.cpsa.ca/wp-content/uploads/2017/02/USRestricted.02142017.pdf. Accessed 19 Aug 2019

32. Chawla TP, Cresswell M, Dhillon S et al (2019) Canadian association of radiologists position statement on point-of-care ultrasound. Can Assoc Radiol J 70(3):219-225. https://doi.org/10.1016/j.carj.2019.06.001

33. Denton C, Iradukunda D, Trickovic J, Turnquist A, Olszynski P. ED Ultrasound in Saskatoon: a quality assurance study; 2019. https://sasksonic. files.wordpress.com/2019/03/wecoc-edus-poster.pdf. Accessed 30 Mar 2019

\section{Publisher's Note}

Springer Nature remains neutral with regard to jurisdictional claims in published maps and institutional affiliations. 